\title{
Das KwEG als Wegbereiter des autoritären Ständestaates
}

\author{
Legislation without Parliament- \\ The use of the 'Wartime Economy Enabling Act' (1917) between March 1933 and May 1934 \\ In March 1933, the Austrian National Parliament became incapable of acting. The government under Federal \\ Chancellor Engelbert Dollfuß announced it would from now on rule on the basis of the 'Wartime Economy Enabling \\ Act', an emergency law passed in 1917. In effect, Austria turned into a dictatorship. Until the proclamation of the \\ authoritarian 'May Constitution' on 1 May 1934, the Government substituted regular legislation with almost 500 \\ 'emergency ordinances' - touching all aspects of life.
}

Keywords: Austrian Civil War 1934 - Austrian History 1933-1934 -

Austrofacism - Engelbert DOLLFUß - Wartime Economy Enabling Act

\section{I.}

Nach der Lahmlegung des Nationalrates Anfang März 1933 herrschte die Regierung Dollfuß mit Hilfe des KwEG weiter; argumentiert wurde mit der „Abwehr der mit einer Störung der öffentlichen Ruhe, Ordnung und Sicherheit verbundenen wirtschaftlichen Gefahren“. Schon die erste Verordnung, die sie erließ - eine zensurähnliche Maßnahme -, ging weit über den Rahmen des Gesetzes hinaus. In den folgenden 14 Monaten wurden 471 auf dem KwEG beruhende Verordnungen in den verschiedensten Bereichen erlassen. Sie betrafen Eingriffe in die Geschworenengerichtsbarkeit ebenso wie die Einrichtung eines Fonds zur Förderung des Straßenbaus, Maßnahmen gegen die Ausbeutung Kreditsuchender oder die Verlängerung des Betriebes des Zahlenlottos.

Die KwEG-Verordnungen dienten zur Auflösung politischer Parteien ebenso wie zur Einrichtung des Österreichischen Gewerkschaftsbundes und griffen massiv in die österreichische Verfassung ein. Der Missbrauch kulminierte in der Ausschaltung der Verfassungsgerichtsbarkeit und endete mit der Einführung der ständisch-autoritären Verfassung vom Mai 1934 ebenfalls im Verordnungswege.

\section{II.}

Die erste auf dem KwEG basierende Verordnung wurde am 7. März 1933 erlassen und bereits einen Tag später im Bundesgesetzblatt kundgemacht. Sie betraf „,besondere Maßnahmen zur Hintanhaltung der mit einer Störung der öffentlichen Ruhe, Ordnung und Sicherheit verbundenen Schädigungen des wirtschaftlichen Lebens ${ }^{\prime \prime} .{ }^{1}$ Dies wurde im Titel der Verordnung ausdrücklich genannt und in der Präambel unter Berufung auf das KwEG mit ähnlichem Wortlaut wiederholt (,,zur Abwehr der mit einer Störung der öffentlichen Ruhe, Ordnung und Sicherheit verbundenen wirtschaftlichen Gefah-

\footnotetext{
${ }^{1}$ BGBl. 41/1933.
} 
ren“). Die Verordnung ermöglichte die Vorlage von Zeitungspflichtstücken zwei Stunden vor dem Beginn der Verbreitung, wenn die Zeitung zuvor wegen eines durch den Inhalt begangenen strafbaren Inhalts gerichtlich beschlagnahmt worden war. Bei Zuwiderhandeln wurden harte Konsequenzen bis zum Verlust der Gewerbeberechtigung angedroht. In Ergänzung zu den zensurähnlichen Maßnahmen wurde in $\S 6$ der Verordnung die öffentliche Beleidigung der Bundesregierung, einer Landesregierung, einer ausländischen Regierung sowie einzelner Regierungsmitglieder zusätzlich zu einer allfälligen strafrechtlichen Konsequenz unter Verwaltungsstrafe gestellt, wenn „durch die Tat die zur Wiederaufrichtung des wirtschaftlichen Lebens unentbehrliche öffentliche Ruhe, Ordnung und Sicherheit gefährdet wird“. Eine Berufungsmöglichkeit gegen Strafbescheide aufgrund der Verordnung wurde bei geringeren verhängten Strafen ausgeschlossen.

Im Ministerrat wurde auch über die Einführung dieser Regelungen mittels einer Notverordnung gemäß Art. 18 B-VG nachgedacht, da auch strafrechtliche Bestimmungen enthalten waren. Wegen der dafür notwendigen Einbindung des Nationalrates - der dafür zuständige Unterausschuss des Hauptausschusses hätte erst eingesetzt werden müssen - verwarf man aber diese Idee. ${ }^{2}$

Zwei Tage später teilte Heeresminister Carl Vaugoin im Ministerrat mit, dass die Christlichsoziale Partei bereit gewesen wäre, eine Sitzung des Hauptausschusses zur Bildung des Ständigen Unterausschusses zu ermöglichen; die zwischenzeitig erfolgte Einberufung einer Sitzung des Nationalrates durch den großdeutschen Abgeordneten Sepp Straffner habe das aber geändert. Offensichtlich war die Einsetzung dieses Unterausschusses dem Landbund ein Anliegen

\footnotetext{
2 Enderle-Burcel, Protokolle Ministerrat VIII/2, Nr. 851, 393 f.
}

gewesen, um neben den KwEG-Verordnungen auch Notverordnungen nach Art. 18 B-VG erlassen zu können. Vizekanzler Franz Winkler ging es dabei um die Umwandlung des Bundesrates in einen Länder- und Ständerat, was durch die B-VG-Novelle 1929 vorgesehen worden war. ${ }^{3}$

Die nächsten Verordnungen auf Basis des KwEG ergingen am 12. März 1933. Sie betrafen alle im weiteren Sinne Finanzangelegenheiten und wären in dieser oder ähnlicher Form wohl auch vom Parlament erlassen worden, da die meisten von ihnen bereits als Regierungsvorlage im Nationalrat eingebracht worden waren. ${ }^{4}$ Erwähnung verdient die mit Verordnung erlassene "Gewerbenovelle 1933“. ${ }^{5}$ Sie nahm zahlreiche Änderungen der Gewerbeordnung und zwei kleinere Anpassungen von Nebengesetzen vor. Diese Verordnung ist die einzige, die keine Präambel mit Berufung auf das KwEG ${ }^{6}$ enthält. Dies dürfte aber ein redaktionelles Versehen sein, da in der Beratung im Ministerrat die Rechtsgrundlage ganz klar war. ${ }^{7}$

Manche Verordnungen beriefen sich nur auf das KwEG, andere nahmen explizit Bezug auf die Abwehr wirtschaftlicher Gefahren. Die Vermutung, dass dies bei Verordnungen, die "politische" Themen betrafen, zur Anwendung kam, geht ins Leere, wie etwa die beiden Wehrgesetznovellen zeigen. ${ }^{8}$

In der Folge wurden jedenfalls verschiedene Präambeln verwendet, wie etwa (sehr häufig) „zur Abwehr der mit einer Störung der öffentlichen Ruhe, Ordnung und Sicherheit verbunde-

\footnotetext{
${ }^{3}$ ENDERLE-BurCel, Protokolle Ministerrat VIII/2, Nr. 853, 417.

${ }^{4}$ Enderle-Burcel, Protokolle Ministerrat VIII/2, Nr. 855, 432ff.

${ }^{5}$ BGBl. 62/1933.

6 "Auf Grund des Gesetzes vom 24. Juli 1917, R.G.Bl. Nr. 307, wird verordnet, wie folgt:"

${ }^{7}$ ENDERLE-Burcel, Protokolle Ministerrat VIII/2, Nr. 855, TOP 13, 435ff.

${ }^{8}$ BGBl. 62 und 67/1933.
} 
nen wirtschaftlichen Gefahren“.9 Andere Präambeln nahmen auf konkrete Anlassfälle Bezug, etwa „zur Abwehr der mit der Durchführung von Wahlen während der Fremdensaison verbundenen wirtschaftlichen Schädigungen", 10 oder „zur Abwehr wirtschaftlicher Schädigungen, die durch die finanziellen Schwierigkeiten der österreichischen Privatbahnunternehmungen verursacht werden". ${ }^{11}$ In einem Fall - den Brennstoffverordnungen" - bezog man sich nicht nur auf das KwEG, sondern auch auf das Brennstoffgesetz. ${ }^{12}$ Die meisten Verordnungen wurden von der Bundesregierung erlassen, einige von einzelnen Bundesministern, manche davon im Einvernehmen mit Regierungskollegen.

Der Ablauf des Zustandekommens der Verordnungen wurde bereits im Ministerrat am 10. März 1933 festgelegt: ${ }^{13}$ Die im Ministerrat vorbesprochenen Vorschläge sollten, versehen mit einem Gutachten des Verfassungsdienstes des Bundeskanzleramtes ${ }^{14}$ über die verfassungsrechtliche Zulässigkeit der geplanten Verordnungen, in einem der drei (formlosen) Ausschüsse für verfassungsrechtliche, wirtschaftspolitische sowie sozialpolitische Angelegenheiten vorberaten werden. ${ }^{15}$ Anschließend sollte der Ministerrat die Verordnung beschließen. Diese Ausschüsse, die aus Vertretern der Regierungsparteien bestanden, tagten bis in den Herbst 1933; ${ }^{16}$ in den allermeisten Fällen wurden die Verordnungsentwürfe aber im Ministerrat eingebracht und ohne weitere Beratung be-

\footnotetext{
${ }_{9}^{9}$ BGBl. 185/1933.

${ }^{10}$ BGB1. 172/1933.

11 BGBl. 235/1933.

12 BGBl. 401/1931.

${ }^{13}$ ENDERLE-BuRCEL, Protokolle Ministerrat VIII/2, Nr. 854, TOP 1, 425.

${ }^{14}$ Auf dieses wurde laut HuEMER, Sektionschef Hecht 319 , ,gelegentlich auch verzichtet“.

${ }^{15}$ ENDERLE-BURCEL, Protokolle Ministerrat VIII/2, Nr. 853, 423.

${ }^{16}$ Goldinger, Protokolle Klubvorstand 12ff.
}

schlossen, was ob der großen Menge nicht verwundert.

Betrachtet man die Verordnungsgebung der folgenden Monate bis zum Herbst 1933, sieht man, dass die Regierung keinem Masterplan folgte, sondern auf die jeweilige Situation reagierte. So erließ die Bundesregierung beispielsweise am 5. April 1933 eine Verordnung betreffend Beschränkungen des Handels mit ausländischen Wertpapieren. ${ }^{17}$ Anlass war, dass in den Wochen davor ein schwunghafter Handel mit Schweizer Bundesbahnobligationen eingesetzt hatte, der eine Gefahr für die Bewertung des Schillings mit sich brachte. ${ }^{18}$

Die Rechtschöpfung erfolgte sehr schnell, Diskussionen fanden oft nicht statt. Da man in vielen Fällen nicht auf Vorarbeiten zurückgreifen konnte, kam es immer wieder zu Ungenauigkeiten bzw. erwiesen sich die Regelungen als nicht zweckmäßig. Durch die oft anlassbezogene Aneinanderreihung punktueller Regelungen mussten zudem nachträglich Änderungen bereits erlassener Verordnungen vorgenommen werden. ${ }^{19}$

Zuweilen kam es im Ministerrat zu Auseinandersetzungen hinsichtlich der Reichweite der Maßnahmen. Dies betraf insbesondere das Vorgehen gegen die politischen Gegner: gegenüber den Kommunisten war man sich einig, während die Härte des Vorgehens gehen die Nationalsozialisten nicht unumstritten war. So stand das Verbot der NSDAP und des Steirischen Heimatschutzes im Juni 1933 außer Streit; die Aberkennung der Mandate ging aber einigen Regierungsmitgliedern zu weit. ${ }^{20}$ Hinsichtlich der Einführung der Todesstrafe im ordentlichen Verfahren lehnte die Regierung den Eingriff in

\footnotetext{
17 BGBl. 105/1933.

${ }^{18}$ Enderle-BurCel, Protokolle Ministerrat VIII/3, Nr. 864, TOP 2, 111 f.

${ }^{19}$ HUEMER, Sektionschef Hecht 320.

${ }^{20}$ DORNER-BRADER, Protokolle Ministerrat VIII/4, Nr. 884 vom 19. 6. 1933, TOP 1, 32.
} 
die Verfassung dezidiert ab, zumal sie befürchtete, dass kein Gericht auf Basis einer solchen Verordnung ein Todesurteil verhängen würde. ${ }^{21}$

Die Problematik des verfassungsmäßigen Rahmens der Verordnungstätigkeit war den Regierungsmitgliedern sehr wohl bewusst. So erwähnte Justizminister Kurt Schuschnigg etwa anlässlich der Anordnung der Anzeigepflicht von Versammlungen „der Vollständigkeit halber" die Bedenken des Verfassungsdienstes des Bundeskanzleramtes und der zuständigen Fachabteilung des Justizministeriums, die er aber nicht teile. ${ }^{22}$

Nach der Trabrennplatzrede vom 11. September 1933, in der Bundeskanzler Engelbert Dollfuß die Abkehr vom Parlamentarismus hin zu einem autoritären Ständestaat angekündigt hatte, erfolgte eine entsprechende Umbildung der Regierung. Die programmatische Änderung schlug sich naturgemäß in den KwEG-Verordnungen nieder, ${ }^{23}$ zudem einige "gemäßigtere" Minister aus der Regierung ausgeschieden waren. ${ }^{24}$

Die Maßnahmen gegen die politischen Gegner, insbesondere die Nationalsozialisten, verbunden mit den gesellschaftspolitischen Weichenstellungen überschritten immer wieder den verfassungsrechtlichen Rahmen, den das KwEG vorgab. Nach dem Februaraufstand 1934 wurde mit der Ausschaltung der Sozialdemokratie auf allen Ebenen und der völligen Zerschlagung der Partei die Demontage des parlamentarischen

\footnotetext{
${ }^{21}$ Vgl. z.B. Finanzminister Karl Buresch, DORNERBRADER, Protokolle Ministerrat VIII/4, Nr. 884 vom 19. 6. 1933, TOP 1, 25.

22 ENDERLE-BURCEL, Protokolle Ministerrat VIII/2, Nr. 856, TOP 5, 453; BGBl. 1933/55.

23 Siehe dazu die entsprechende Ankündigung des Bundeskanzlers in der ersten Sitzung der neuen Regierung, DORNER-BRADER, Protokolle Ministerrat VIII/4, Nr. 898 vom 22. 9. 1933, TOP 1, 387.

${ }^{24}$ So hatte sich etwa Vizekanzler Franz Winkler vehement gegen die Einrichtung von Anhaltelagern ausgesprochen; DORNER-BRADER, Protokolle Ministerrat VIII/4, Nr. 896, TOP 21, 334 sowie Nr. 897, TOP 15, $367 f$.
}

Systems in die abschließende Phase geführt. Die Regierung arbeitete von nun an intensiv und zügig auf die Errichtung des neuen Herrschaftssystems hin. Den Höhe- und Schlusspunkt der verfassungsrechtlichen Farce bildet die Einführung der neuen Verfassung: Am 24. April 1934 erließ die Regierung die Verordnung „über die Verfassung des Bundesstaates Österreich“. Die Bestimmungen der ,in der Anlage kundgemachten Verfassungsurkunde" sollten fortan die neue Verfassung sein. ${ }^{25}$ Der Beginn war durch ein besonderes Bundesverfassungsgesetz $\mathrm{zu}$ bestimmen, welchen das am 30. April 1934 wieder einberufene (Rumpf-)Parlament beschloss. Am 1. Mai erfolgte die erneute Kundmachung der Verfassung durch die Bundesregierung.

\section{III.}

Die auf Basis des KwEG erlassenen Verordnungen lassen sich im Groben in fünf inhaltliche Themenbereiche gliedern:

1. Aufrechterhaltung der Herrschaft

2. Gesellschaftspolitische Änderungen

3. Sanierung des Staatshaushalts

4. Wirtschafts- und Sozialpolitik

5. "Allgemeines"

\section{Aufrechterhaltung der Herrschaft}

$\mathrm{Zu}$ diesem Block zählt der Großteil der KwEGVerordnungen, weshalb nur einige wenige beispielhaft herausgegriffen werden können.

Als deutlichster Bruch der Verfassung ist wohl die Lahmlegung des Verfassungsgerichtshofes zu nennen. Mit der Verhinderung der Prüfmöglichkeit von Verordnungen im Wege einer selbst verfassungswidrigen Verordnung beging die Regierung im Mai wohl einen der massivsten

${ }^{25}$ BGBl. I 239/1934. 
Verstöße gegen den Rechtsstaat. ${ }^{26}$ Anlassfall war die Anfechtung mehrerer Verordnungen der Bundesregierung durch die Wiener Landesregierung beim Verfassungsgerichtshof; weitere Anfechtungen folgten. Die Sache drängte schließlich so sehr, dass die Regierung die Verordnung einen Tag vor dem nächsten Ministerrat im Umlauf beschloss. ${ }^{27}$

Zumindest in einem Fall hätte die Regierung den Verfassungsgerichtshof im Übrigen gebraucht: Am 26. Februar 1934 beschloss der Salzburger Landtag ein Verfassungsgesetz, in dem er der Landesregierung seine Gesetzgebungskompetenz übertrug. ${ }^{28}$ Solche Verordnungen sollten wirksam werden, wenn das zuständige Bundesministerium nicht binnen zwei Wochen Einspruch erhob, was den bisherigen Einspruchsrechten bei Landesgesetzen über Landes- und Gemeindeabgaben widersprach. Da der Verfassungsgerichtshof nicht eingreifen konnte, schob die Bundesregierung dem im Wege einer allgemeinen Regelung den Riegel vor. ${ }^{29}$ Der Bundeskanzler hielt in diesem $\mathrm{Zu}$ sammenhang fest, die Verordnung ,mache auch insofern keinen ungünstigen Eindruck, als sie zur Wahrung der bestehenden Verfassung erlassen werde $[\ldots]^{\prime \prime} .{ }^{30}$

Besonders schwerwiegend und rechtsstaatlich bedenklich waren die zahlreichen Eingriffe in

\footnotetext{
${ }^{26}$ BGBl. 191/1933. Dazu eingehend POLASCHEK, Rechtsentwicklung 100f.

${ }^{27}$ HuEMER, Sektionschef Hecht, 183.

${ }^{28}$ LGBl. 47/1934, Art. 3.

29 „Verordnung [...] betreffend das Einspruchsrecht der Bundesregierung auf Grund des Finanzverfassungsgesetzes", BGBl. I 159/1934. Die Verordnung galt nicht für Wien, was mit BGBl. I 215/1934 geändert wurde. Siehe auch die Verordnung über das Erfordernis der Zustimmung der Bundesregierung zur Kundmachung von Verordnungen der Länder (außer Wien) womit landesverfassungsgesetzliche Bestimmungen abgeändert werden, BGBl. I 161/1934.

${ }^{30}$ ENDERLE-BurCel, Protokolle Ministerrat VIII/2, Nr. 928 vom 9. 3. 1934, TOP 12, S99ff.
}

das Straf- und Strafprozessrecht. ${ }^{31}$ Die Verhängung des Standrechtes sowie die Ausdehnung der Straftatbestände, die in diesem Verfahren mit der Todesstrafe geahndet werden konnten, richteten sich in erster Linie gegen nationalsozialistische Terroranschläge. De facto wurden diese Maßnahmen aber auch gegen andere politische Gegner („Februaraufstand“) sowie gegen „unpolitische“ Straftäter eingesetzt. ${ }^{32}$ Auch die Einschränkung der Geschwornengerichtsbarkeit und die Unterminierung der Unabhängigkeit der Richter ${ }^{33}$ dienten in erster Linie dem Kampf gegen die Nationalsozialisten.

Auch im Verwaltungsstrafrecht kam es zu einigen grundlegenden Verschärfungen. So wurde für alle KwEG-Verordnungen die für die Zulässigkeit der Berufung gegen Strafbescheide der Verwaltungsbehörden erforderliche Strafhöhe von 200 Schilling Geldstrafe auf 1.000 Schilling sowie von 14 Tagen Arrest auf sechs Wochen Arrest erhöht. ${ }^{34} \mathrm{Um}$ die rasche Vollstreckung von Verwaltungsstrafen zu gewährleisten, konnte Beschwerden an den Verwaltungsgerichtshof die aufschiebende Wirkung aberkannt werden, wenn „öffentliche Rücksichten die sofortige Vollstreckung gebieten“. ${ }^{35}$

Um Proteste gegen die Regierung zu verhindern, wurden das Versammlungsrecht und das Streikrecht massiv eingeschränkt. ${ }^{36}$

\footnotetext{
${ }^{31}$ Beginnend mit der Verordnung des Bundesministers für Justiz vom 24. 3. 1933, „womit zur Hintanhaltung der mit einer Störung der öffentlichen Ruhe, Ordnung und Sicherheit verbundenen Schädigungen des wirtschaftlichen Lebens besondere Maßnahmen für das Verfahren vor den Geschwornengerichten getroffen werden“, BGBl. 81/1933.

32 POLASCHEK, Todesstrafe 41f.

${ }^{33}$ BGBl. I 83/1934.

${ }^{34}$ BGBl. 237/1933.

35 „1. Verwaltungsgerichtshofgesetznovelle”, BGB1. 324/1933, Art. II. Eine solche Novelle war bereits im Jänner geplant und im Nationalrat eingebracht worden; ENDERLE-BurCel, Protokolle Ministerrat VIII/2, Nr. 841, TOP 4, 212.

${ }^{36}$ BGBl. 55 und 138/1933.
} 
Am 13. Juni 1933 wurden die Sicherheitsagenden auf Landesebene den Landeshauptmännern entzogen. Stattdessen wurden Sicherheitsdirektoren eingesetzt, welche unmittelbar dem Bundeskanzler unterstanden. ${ }^{37}$

Einen massiven Eingriff in das Recht der persönlichen Freiheit brachte die Verordnung vom 23. September 1933 ,betreffend die Verhaltung sicherheitsgefährlicher Personen zum Aufenthalte in einem bestimmten Orte oder Gebiete". ${ }^{38}$ Dadurch konnte man auch ohne Vorliegen einer strafbaren Handlung präventiv Personen in "Anhaltelagern" unterbringen. ${ }^{39}$ Mit Verordnung vom 7. Juli $1933^{40}$ wurden "Terrorakte“ (damit waren vor allem Sprengstoffanschläge gemeint) auch unter Verwaltungsstrafe gestellt, um so rascher gegen die Täter vorgehen zu können. ${ }^{41}$ Um eine unmittelbare Vollstreckung der Strafe zu gewährleisten, wurde Berufungen die aufschiebende Wirkung aberkannt und ein Strafaufschub generell für unzulässig erklärt.

Weitere Regelungen in diesem Zusammenhang betrafen das Verbot von Wahlen auf allen Ebenen. Nachdem die NSDAP bei den Gemeinderatswahlen in Innsbruck am 23. April 1933 mit $41,1 \%$ die stärkste Partei geworden war, und die Landtagswahlen in Tirol ein ähnliches Ergebnis befürchten ließen, verschob die Landesregierung diese auf unbestimmte Zeit. Wenn auch keine weiteren Wahlen anstanden, befürchtete man, dass solche durch Mandatsniederlegungen erzwungen werden könnten. ${ }^{42}$ Mit Verordnung vom 10. Mai 1933 betreffend die

\footnotetext{
${ }^{37}$ BGBl. 226/1933. Dabei wurde auf das KwEG „im Sinne des Art 102 Abs 2 B-VG" Bezug genommen.

${ }^{38}$ BGB1. 431/1933. Zu dieser Verordnung BAUER, Österreichische Anhaltelager 13ff.

${ }^{39}$ Die Verordnung sollte mit 1.10.1934 außer Kraft treten.

${ }^{40}$ BGBl. 295/1933.

${ }^{41}$ DORNER-BRADER, Protokolle Ministerrat VIII/4, Nr. 889, Beilage E zu TOP 7, 173.

${ }^{42}$ ENDERLE-BurCel, Protokolle Ministerrat VIII/3, Nr. 872, TOP 10, 292 f.
}

Ausschreibung von Wahlen in Landtage und Ortsgemeindevertretungen - „zur Abwehr der mit der Durchführung von Wahlen während der Fremdensaison verbundenen wirtschaftlichen Schädigungen" - durften bis 31. Oktober 1933 keine solchen Wahlen ausgeschrieben werden bzw. wurden bereits erfolgte Ausschreibungen für nichtig erklärt. ${ }^{43}$ Dieses Verbot wurde im Oktober 1933 bis 31. März 1934 und im März 1934 bis 31. Dezember 1934 verlängert. ${ }^{44}$

Am 26. Mai 1933 wurde die Kommunistische Partei verboten, am 19. Juni die NSDAP und der Steirische Heimatschutz (Führung Kammerhofer). ${ }^{45}$ Einen Monat später folgte die Verordnung über die Beschlagnahme und den Verfall des Vermögens verbotener politischer Parteien. ${ }^{46}$

Nach dem Februaraufstand wurde auch die Sozialdemokratische Partei verboten und ihre Mandate aberkannt. ${ }^{47}$ Damit einher ging eine Zerschlagung des politischen und wirtschaftlichen Bereiches, der rund um diese Partei entstanden war, ${ }^{48}$ sowie die zeitweilige Unterstellung der Verwaltung Wiens unter einen Bundeskommissär. ${ }^{49}$

Die erste Verordnung auf Grund des KwEG betraf (unter anderem) die Kontrolle der Medien, ${ }^{50}$ und auch in der Folge ergriff die Regierung verschiedenste Maßnahmen, mit denen sie gegen regierungskritische Propaganda, insbesondere durch die Nationalsozialisten, vorging. Dies betraf nicht nur Druckschriften, sondern auch Plakate und Flugblätter ${ }^{51}$ sowie ausländische Rundfunksendungen ${ }^{52}$ und Kinofilme. ${ }^{53}$

\footnotetext{
43 BGBl. 172/1933.

${ }^{44}$ BGBl. 476/1933 und BGBl. I 182/1934.

${ }^{45}$ BGBl. 200 bzw. 240/1933.

${ }^{46}$ BGBl. 368/1933 vom 16. 8. 1933.

${ }^{47}$ BGBl. I 78/1934.

${ }^{48}$ Insbesondere BGBl. I 98 bis 104/1934.

${ }^{49}$ BGBl. I 77/1934.

${ }^{50}$ BGBl. 41/1933.

${ }^{51}$ BGBl. 155/1933.

52 BGBl. 300/1933.

${ }^{53}$ BGBl. I 204/1934.
} 
Die Regierung tat sich allerdings schwer, der Provokationen durch die Nationalsozialisten Herr zu werden. Um die Erregung öffentlichen Ärgernisses und ähnliche Verwaltungsstraftaten besser verfolgen $\mathrm{zu}$ können, wurden diese, wenn sie "als politische Demonstration" begangen wurden, unter eine empfindlich höhere Strafe gestellt. ${ }^{54}$

Auch der öffentliche Gebrauch von Fahnen, Flaggen, Wimpeln usw. wurde untersagt, wenn dadurch die öffentliche Ruhe, Ordnung und Sicherheit gefährdet wurden. Dies galt „ausnahmslos für den öffentlichen Gebrauch von roten Fahnen [...], solchen mit dem Sowjetstern, solchen mit den drei Pfeilen und solchen mit dem Hakenkreuz". Für den öffentlichen Gebrauch sonstiger Flaggen usw., „durch die eine parteipolitische Einstellung zum Ausdruck gebracht wird“, war eine Bewilligung des Bundeskanzleramtes einzuholen. ${ }^{55}$ Ebenfalls unter Verwaltungsstrafe gestellt wurde das Anbringen von Bildern, Zeichen usw. „politischer Tendenz" an fremdem Eigentum sowie das Ausstreuen solcher Symbole. Neben der Strafe konnte auch den Tätern gleich der Ersatz des eingetretenen Schadens bzw. der Kosten für die Entfernung der Gegenstände auferlegt werden. Wurde die Tat durch Organe einer politischen Partei begangen, haftete auch diese. ${ }^{56}$

Auf Grund einer weiteren Verordnung konnten die Kosten für außerordentliche Sicherheitsmaßnahmen gegenüber Personen, "die durch strafbares Verhalten diese Sicherheitsmaßnahmen verursacht haben sowie denjenigen, die dieses Verhalten begünstigt oder gefördert haben", eingefordert werden. Personen, die aufgrund der zuvor genannten Verordnung zur Kostenersatzleistung verpflichtet waren, konn-

\footnotetext{
${ }^{54}$ BGBl. 185/1933, betreffend Verwaltungsübertretungen gem. Art. VIII Abs. 1 lit. a, b oder c EGVG, BGBl. 273/1925.

${ }^{55}$ BGBl. 186/1933, „Fahnenverordnung“.

${ }^{56}$ BGBl. 248/1933.
}

ten nun auch „verhalten werden, die verbotswidrig angebrachten oder ausgestreuten Gegenstände persönlich zu entfernen ". ${ }^{57}$

Da ein guter Teil der höheren Beamtenschaft großdeutsch eingestellt und mit dem Regierungskurs nicht einverstanden war, wurde das Disziplinarrecht massiv verschärft. Aufgrund der Verordnung vom 10. Mai 1933 über besondere Maßnahmen betreffend die öffentlichrechtlichen Bundesangestellten ${ }^{58}$ hatten diese erneut den Diensteid abzulegen; die Verweigerung galt als Kündigung (bzw. im Heer als Entlassungsgrund). Eine Entlassung konnte unter anderem ausgesprochen werden, wenn jemand seine Dienstpflichten dadurch verletzte, „dass er geflissentlich staats- oder regierungsfeindliche Bestrebungen fördert" oder andere $\mathrm{zu}$ "politischen" strafbaren Handlungen zu verleiten sucht. Außerdem wurde eine besondere Disziplinarkommission eingesetzt, gegen deren Entscheidungen kein ordentliches Rechtsmittel möglich war.

Solche Eingriffe betrafen auch andere "staatsnahe" Bereiche wie Schulen, Kammern, Sozialversicherungsträger usw. ${ }^{59}$ Verordnungen wie jene vom 13. September 1933 für das Land Steiermark, „womit besondere Maßnahmen gegen staats- und regierungsfeindliche Handlungen von im Dienst- oder Ruhestande befindlichen Lehrkräften an den öffentlichen Volks- und Hauptschulen sowie deren Hinterbliebenen getroffen werden", ${ }^{60}$ ergingen in verschiedener Form und Abstufung bis auf Burgenland, Vorarlberg und Oberösterreich auch für die anderen Bundesländer.

Nach dem Februaraufstand wurde das Bundeskanzleramt zusätzlich ermächtigt, öffentlich

\footnotetext{
${ }^{57}$ BGBl. 397/1933. Siehe dazu auch die Verordnung BGBl. I 20/1934 „über die Ersatzleistung für Schäden aus Terrorakten".

${ }^{58}$ BGBl. 173/1933.

59 Siehe z.B. BGBl. 593/1933, BGBl. I 52/1934.

${ }^{60}$ BGBl. 421/1933.
} 
Bedienstete ,aus Gründen der Aufrechterhaltung der öffentlichen Ruhe, Ordnung und Sicherheit" ihres Dienstes zu entheben und ihre Bezüge bis auf zwei Drittel herabzusetzen. Die Verordnung galt ausdrücklich nicht für Richter und war mit 30. Juni 1934 befristet.61 Private Arbeitnehmer konnten auf Grund einer Verordnung vom 9. März 1934 wegen staatsoder regierungsfeindliche Betätigung entlassen werden. ${ }^{62}$

Zur Unterstützung von Bundesheer, Polizei und Gendarmerie wurden für ersteres „freiwillige Assistenzkörper"63 sowie für die beiden letzteren das "freiwillige" Schutzkorps"64 aufgestellt. Beide sollten sich aus den regierungstreuen Wehrverbänden rekrutieren und im Falle von Unruhen sowie für den Grenzschutz zur Verfügung stehen.

\section{Gesellschaftspolitische Änderungen}

Die Vorstellung der Regierung, insbesondere von Bundeskanzler Dollfuß, einer autoritären, katholisch-konservativen Gesellschaft fand ihren Niederschlag in zahlreichen Verordnungen, welche sich punktuell mit einzelnen Themen befassten.

Nachdem das Tonkinowesen zur Bundessache gemacht worden war, ${ }^{65}$ wurde den Kinobesitzern vorgeschrieben, zur Förderung der österreichischen Tonfilmindustrie sowie „im Dienst der Heimatpropaganda"66 bei allen Aufführungen österreichische Kurzfilme vorzuführen. Diese sollten „hauptsächlich der Verbreitung von Kenntnissen des kulturellen und wirtschaft-

\footnotetext{
${ }^{61}$ BGBl. I 120/1934 vom 23.2.1934.

${ }^{62}$ BGBl. I 157/1934. Diese Verordnung trat ebenfalls am 30. 6. 1934 außer Kraft.

${ }^{63}$ BGBl. 201, 202 und 230/1933.

${ }^{64}$ BGB1. 292/1933 befristet bis 31. 12. 1933; Verlängerung bis 31. 12. 1934 mit BGBl. 576/1933.

${ }^{65}$ BGBl. 131/1933.

${ }^{66}$ ENDERLE-BurCel, Protokolle Ministerrat VIII/3, Nr. 863, TOP 2, 68.
}

lichen Lebens in Österreich und der österreichischen Landschaften sowie der Wiedergabe aktueller Ereignisse aus Österreich dienen".67

Die Verordnung „zum Schutze der Sittlichkeit und Volksgesundheit" richtete sich gegen den öffentlichen Vertrieb von Darstellungen des „ganz oder vorwiegend nackten menschlichen Körpers" in Zeitungen, auf Ansichtskarten oder Fotos. ${ }^{68}$ Außerdem enthielt sie ausführliche Regeln betreffend den Vertrieb empfängnisverhütender Mittel und Medikamente.

Auch im Schulwesen nahm man massive Änderungen vor. Im Gegensatz zur Schulreform 1927 wurden Mittel- und Hauptschulen wieder klar getrennt. In die Hauptschule wurden nur Schüler aufgenommen, „die von der Volksschule zum Aufsteigen in die Hauptschule als reif erklärt" wurden; die übrigen Schüler durften nur die „Abschlussklassen“ der Volksschule, welche den zweiten Klassenzug der Hauptschulen ersetzten, besuchen. ${ }^{69}$ Für die Mittelschulen wurden wieder eigene Aufnahmeprüfungen eingeführt sowie die Übertritte zwischen den Schultypen erschwert. Neben Sparüberlegungen sollte durch eine Einschränkung der Schülerzahlen „dem beängstigenden Anschwellen des gebildeten Proletariates" begegnet werden. ${ }^{70}$ Mädchen sollten nach Möglichkeit in getrennten Schulen unterrichtet werden, zumindest aber in getrennten Klassen. ${ }^{71}$

Der Abbau von (verheirateten) Frauen im öffentlichen Dienst hatte sowohl arbeitsmarktpolitische als auch gesellschaftspolitische Ziele. Zum einen sollten dadurch mehr Männer Arbeit bekommen, zum anderen sollten sich Frauen in

\footnotetext{
${ }^{67}$ Art. II Abs. 1 leg.cit.

${ }^{68}$ BGBl. 219/1933; abgelöst durch Verordnung BGBl. I 171/1934.

${ }^{69}$ BGBl. I 197/1934. Zum Schulwesen TIMISCHL, Beiträge $42 \mathrm{f}$.

70 Bundesminister Otto Ender, DORNER-BRADER, Protokolle Ministerrat VIII/4, Nr. 894 vom 16.8.1933, TOP 8, 282.

${ }^{71}$ BGBl. I 198/1934.
} 
ihren „angestammten“ Bereich von Haushalt und Familie zurückgedrängt werden. ${ }^{72}$

Im Arbeitsrecht kam es zu einer Neuregelung des Kammer-, Betriebsrats- und Gewerkschaftswesens. Mitbestimmungsrechte der Arbeitnehmer wurden in vielen Bereichen soweit wie möglich beschnitten. Dies wie auch die Neuordnung der Konsumgenossenschaften steht natürlich auch in engem Zusammenhang mit der Einschränkung des Machtbereichs der Sozialdemokratie, die nach dem Februaraufstand 1934 schlussendlich völlig zerschlagen wurde.

Am 21. Dezember 1933 wurden in den Bundesbetrieben die Betriebsräte aufgelöst und durch machtlose Personalvertretungen ersetzt; ${ }^{73}$ gleichzeitig wurden in den Arbeiterkammern Verwaltungskommissionen eingesetzt, wodurch alle regierungskritischen Funktionäre abgelöst wurden. ${ }^{74}$

Am 2. März 1934 wurde der "Gewerkschaftsbund der österreichischen Arbeiter und Angestellten“ ins Leben gerufen, um - so die Präambel - „im Geiste des Christentums, der sozialen Gerechtigkeit und Liebe zum Vaterlande den Arbeitern und Angestellten eine wirksame Interessenvertretung zu sichern und ihre Eingliederung in den berufsständischen Aufbau der Gesellschaft vorzubereiten“ ${ }^{75}$

\footnotetext{
${ }^{72}$ Siehe etwa die Verordnung der Bundesregierung „über den Abbau verheirateter weiblicher Personen im Bundesdienste und andere dienstrechtliche Maßnahmen", BGB1.545/1933, die Verordnungen des Bundesministers für Unterricht „wirksam für das Land Salzburg, über den Abbau der verheirateten Lehrerinnen“ sowie „wirksam für Oberösterreich, betreffend den Abbau der verheirateten Lehrerinnen an Volks- und Hauptschulen", BGBl. I 36 und $178 / 1934$

${ }^{73}$ BGB1. 571/1933.

${ }^{74}$ BGBl. 572/1933.

${ }^{75}$ BGBl. I 132/1934; TALOS, Austrofaschistische Herrschaftssystem $336 f$.
}

Ihren Höhepunkt fanden alle diese Maßnahmen natürlich in der Einführung der neuen Verfassung. Dazu wurde als erstes die Geschäftsordnung des Nationalrates dahingehend abgeändert, dass beim Rücktritt aller drei Präsidenten bis zur Wahl eines neuen Präsidenten jener Abgeordnete den Vorsitz übernimmt, der der stärksten im Nationalrat vertretenen Partei angehört und Mitglied des früheren Präsidiums war. ${ }^{76}$

Die Verordnung „über die Verfassung des Bundesstaates Österreich“ vom 24. April 1934 enthielt in ihrer Anlage die neue "Verfassungsurkunde“.77 Der "Beginn der Wirksamkeit“ der Bestimmungen sollte durch ein eigenes Bundesverfassungsgesetz bestimmt werden. Dieses wurde am 30. April vom wiedereinberufenen Rumpfparlament beschlossen, dem nach der Aberkennung der Mandate der Sozialdemokratischen Partei nur mehr 91 Abgeordnete angehörten. ${ }^{78}$ Am 1. Mai 1934 erfolgte durch die Bundesregierung eine erneute Kundmachung der Verfassung. ${ }^{79}$ Schon am 27. April hatte die Regierung „zum dauernden Gedenken an die Proklamation der Verfassung 1934" den 1. Mai als allgemeinen Ruhe- und Festtag erklärt. ${ }^{80}$

\section{Finanzierung des Staates}

Die schlechte Finanzsituation des Staates verbunden mit dem Bedarf nach höheren Ausgaben im Sicherheitsbereich führte zu radikalen Einsparungsmaßnahmen. Dazu zählten etwa eine Senkung des Aufwandes bei den Sozialversicherungsträgern, ${ }^{81}$ Kürzungen der Arbeitslosenun-

\footnotetext{
${ }^{76}$ BGBl. I 2381/934 vom 24. 4. 1934.

77 BGBl. I 239/1934.

${ }^{78}$ BGBl. I 255/1934; POLASCHEK, Rechtsentwicklung, $36 \mathrm{ff}$.

${ }^{79}$ BGBl. II 1/1934.

${ }^{80}$ BGBl. I 249/1934.

${ }^{81}$ So wurden beispielsweise für die Bediensteten der Industriellen Bezirkskommissionen und Arbeitslosenämter die Urlaubs- und Weihnachtsremunerationen gestrichen; BGBl. 123/1933.
} 
terstützung, ${ }^{82}$ die Erhöhung von Steuern ${ }^{83}$ und die Einführung neuer Abgaben. ${ }^{84}$

Zahlreiche Sonderregelungen betrafen den Schulbereich. Dabei ging es etwa um Maßnahmen zur Verringerung der Lehrerstellen an den öffentlichen Volks- und Hauptschulen in Niederösterreich, wodurch 600 Stellen gestrichen wurden. ${ }^{85}$ Es wurden aber auch teilweise Kürzungen des Diensteinkommens von Lehrern (Vorarlberg, Salzburg) ${ }^{86}$ oder der Remunerationen für die klassenweise Erteilung des Religionsunterrichtes (Niederösterreich) vorgenommen. ${ }^{87}$

Besonders hohe Kosten verursachte der Bahnbetrieb. Deshalb wurde im März 1933 die Auszahlung der Bezüge der ÖBB-Bediensteten und -Pensionisten „zwecks Hintanhaltung wirtschaftlicher Schädigung" in zwei, bei finanziellen Engpässen vorübergehend auch drei Monatsraten ermöglicht. ${ }^{88}$ Weitere Einsparungen erfolgten durch die Bundesbahnbudgetsanierungsverordnung vom 10. April 1933; sie erging explizit „zur Abwehr wirtschaftlicher Schädigungen die durch die dem Bunde obliegende Bedeckung des Abgangs im Haushalte der Unternehmung ,Österreichische Bundesbahnen'

${ }^{82}$ Durch die Verordnung ,"über die zeitweilige Abänderung des Ausmaßes der Arbeitslosenunterstützung", BGBl.343/1933, die vom 6.8.1933 bis 31. 12. 1934 gelten sollte, sollten beispielsweise 30 Millionen Schilling eingespart werden; DORNERBRADER, Protokolle Ministerrat VIII/4, Nr. 893, TOP 9, 256.

${ }^{83}$ Z.B. BGBl. 189/1933 über die Erhöhung der Süßstoffsteuer.

${ }^{84}$ Z.B. die "Zigarettenhüllenabgabeverordnung" BGB1. 337/1933.

${ }^{85}$ BGB1. 278/1933.

${ }^{86}$ BGBl. 599/1933 bzw. BGBl. 40/1934.

${ }^{87}$ BGBl. I 110/1934.

${ }^{88}$ BGBl. 72/1933. Diese beabsichtigte Maßnahme hatte Anfang März zum Warnstreik der Eisenbahner geführt, der zur Eskalation der Nationalratssitzung am 4.3. 1933 geführt hatte. Die Verordnung galt bis 31. 12.1933, wurde aber mit Verordnung BGBl. 564/1933 bis 31. 12. 1935 erstreckt. erwachsen“. ${ }^{89}$ Die Regierungsvorlage für ein entsprechendes Bundesgesetz war bereits Ende Juli 1932 im Nationalrat eingebracht, aber wenig später zurückgestellt worden.90 In Ergänzung dazu wurde die Einhebung eines Preiszuschlages bei Fahrkarten zum Personalfahrpreise und eines Zuschlages bei Freifahrtausweisen eingeführt. ${ }^{91}$

Diese Einsparungsmaßnahmen wurden, ergänzt um weitere Regelungen, am 9. Juni 1933 auch auf Privatbahnen ausgedehnt.92 Am 30. September 1933 wurde mit einer weiteren Verordnung die Verminderung des Personalstandes der ÖBB ohne Einvernehmen mit der Personalvertretung ermöglicht. ${ }^{93}$

Da die Ausgaben zur Aufrechterhaltung der Sicherheit im Frühjahr 1934 ein extremes Ausmaß erreicht hatten, sah man sich Ende März zur Einführung einer außerordentlichen Sicherheitssteuer gezwungen. ${ }^{94}$ In Ergänzung zum Krisensteuergesetz $1931^{95}$ wurde für die Jahre 1934 und 1935 eine zusätzliche Steuer vom Einkommen Lediger sowie von Vermögen erhoben; „mit Rücksicht auf den besonderen Anlass der Einführung dieser Steuer" wurde eine Verlängerung der Geltungsdauer ausdrücklich im Gesetz ausgeschlossen. ${ }^{96}$

Ein in dieser Form einzigartiger Schritt war der Zugriff auf die Finanzen von Wien. Die Stadt hatte für die Jahre 1933 und 1934 einen „Lastenbeitrag“ von je 36 Millionen Schilling zu entrichten. ${ }^{77}$ In 15 Verordnungen wurden dem Stadthaushalt insgesamt 100 Millionen Schilling ent-

\footnotetext{
${ }^{89}$ BGBl. 122/1933.

${ }^{90}$ Enderle-Burcel, Protokolle Ministerrat VIII/3, Nr. 866, TOP 15, 159.

${ }^{91}$ BGBl. 121/1933.

92 BGBl. 235/1933.

${ }_{93}$ BGBl. 453/1933.

${ }^{94}$ BGBl. I 183/1934.

${ }^{95}$ BGBl. 294/1931.

$96 \S 12$ leg. cit.

${ }^{97}$ BGBl. 380/1933. Die Verordnung trat rückwirkend mit 1. 1. 1933 in Kraft und galt bis Ende 1934.
} 
nommen. ${ }^{98}$ Wien verlor dadurch etwa ein Drittel seiner Einnahmen und konnte praktisch keine (sozialdemokratische) inhaltliche Politik mehr machen.

\section{Wirtschafts- und Sozialpolitik}

Neben zahlreichen Einsparungen versuchte die Regierung, die Wirtschaft anzukurbeln beziehungsweise wirtschaftlichen Krisensituationen zu begegnen. Eine sehr kostenintensive Aufgabe war die Sanierung des Bankensektors. Die bei der Nationalbank eingerichtete Stützungsgesellschaft erhielt im März 1933 vom Bund Vermögenschaften im Wert von 140 Millionen;99 im April 1934 übernahm man für die niederösterreichische Industrieholdinggesellschaft eine Ausfallshaftung von bis zu 20 Millionen Schilling. ${ }^{100}$ Um die Kosten der Banken zu senken, wurde ihnen ermöglicht, in Arbeitsverträge und Pensionen einzugreifen. ${ }^{101}$

$\mathrm{Zu}$ den ersten Maßnahmen der Bundesregierung im März 1933 zählte der Schutz des Gewerbes. Mit der „Gewerbenovelle 1933“ wurden unter anderem die Konsumgenossenschaften der Gewerbeordnung unterstellt.102 Mehrere Verordnungen beschränkten den Antritt und die Ausübung von Gewerben, ${ }^{103}$ mit einer weiteren Verordnung wurden "Einheitspreisgeschäfte“ verboten. ${ }^{104}$

Zur Belebung des Arbeitsmarktes griff die Regierung in verschiedenste arbeitsrechtliche Schutzvorschriften ein. Friseure, Fleischhauer und Lebensmittelhändler durften etwa an Feier-

\footnotetext{
${ }^{98}$ ENDERLE-BURCEL, Protokolle Ministerrat VIII/6, XXI m.w.N.

${ }^{99}$ BGB1. 69/1933.

${ }^{100}$ BGBl. I 232/1934 „betreffend Maßnahmen zur Reorganisation von Banken“.

101 „Bankentlastungsverordnung“ BGBl. 68/1933,

„Bankpensionsverordnung“ BGBl.377/1933; TALOS,

Herrschaftssystem, 316f.

102 BGBl. 52/1933.

${ }^{103}$ BGBl. 53, 84, 148, 283, 467/1933.

104 BGBl. 54/1933.
}

tagen von 8 bis 11 Uhr arbeiten, umfangreiche Regelungen betrafen die Bäckereiarbeiter. ${ }^{105}$ "Zur Förderung der Arbeitsbeschaffung und zur Bekämpfung der Arbeitslosigkeit" wurden die Arbeiter bei öffentlichen Bauten von den Kollektivverträgen und Satzungen ausgenommen,106 der 1932 eingeführte freiwillige Arbeitsdienst zur Beschäftigung jugendlicher unqualifizierter Arbeitsloser wurde ausgeweitet. ${ }^{107}$ „Zum Schutze des heimischen Arbeitsmarktes" durfte die Einbürgerung von Ausländern „bis auf weiteres" nur in Einzelfällen erfolgen. ${ }^{108}$ Daneben wurden Arbeitszeitbeschränkungen und Urlaubsregelungen gelockert und Überstundenvergütungen mussten rascher geltend gemacht werden. ${ }^{109}$

Um den Wirtschaftsverkehr auf der Donau anzukurbeln, beschloss die Regierung Steuer- und Gebührenbefreiungen der Bundesbeihilfen für die DDSG, 110 zur Belebung des Fernfrachtverkehrs durch die Bahn schuf man entsprechende Auflagen für den Güterverkehr mit Lastkraftwagen. ${ }^{111}$

Durch die Gründung von Ausfuhrorganisationen, wie beispielsweise den Holzwirtschaftsrat, sollte der Export angekurbelt werden, ${ }^{112}$ parallel dazu wurden Einfuhrverbote verhängt. ${ }^{113}$

Die schlechte Wirtschaftslage spiegelt sich auch in den Unterstützungsmaßnahmen beispielsweise für notleidende Gebirgsbauern, Arbeitslose und Kleinrentner ${ }^{114}$ oder der "Winterhilfe 1933/1934. ${ }^{115}$ Wirte, welche aufgrund der 1000Mark-Sperre massive Umsatzeinbußen hatten,

\footnotetext{
105 BGBl. 262 und 212/1933.

106 BGBl. 225/1933.

107 BGBl. 126 und 229/1933.

${ }^{108}$ BGBl. 523/1933.

${ }^{109}$ BGBl. 209/1933.

${ }^{110}$ BGBl. 85/193.

111 BGB1. 253/1933.

112 BGBl. 180, 287, 406/1933.

113 BGBl. 335 und 367/1933; BGB1. I 22, 188, 226/1934.

114 BGBl. 196/1933.

${ }^{115}$ BGBl. 493/1933.
} 
wurden durch einen Erlass des Pachtzinses unterstützt. ${ }^{116}$

\section{5. „Allgemeines"}

Neben den aufgezählten Maßnahmen, welche zumindest in den ersten beiden Themenbereichen unter „normalen“ politischen Bedingungen nicht oder zumindest nicht in dieser Form durchgeführt worden wären, hatte die Regierung auch Verordnungen zu erlassen, welche sich aus „allgemeinen“ Notwendigkeiten ergaben und auch im parlamentarischen Betrieb eher routinemäßig abgehandelt worden wären.

Dazu zählt etwa die Umsetzung von völkerrechtlichen Verträgen, wie etwa ein Handelsund Schifffahrtsvertrag mit Schweden,117 oder die Erklärung von Straßenzügen als Bundesstraßen. 118

\section{IV.}

Viele der bis zum Sommer 1933 erlassenen Verordnungen basierten auf Vorarbeiten, die vor dem März 1933 vom Nationalrat bereits behandelt worden oder zumindest dafür vorgesehen waren. Eine Sanierung dieser Normen auf parlamentarischem Wege wäre also durchaus denkbar gewesen. ${ }^{119}$ Im Laufe der Zeit wurden aber für die Erlassung von Verordnungen immer stärker innenpolitische und wirtschaftliche Schwierigkeiten als Vorwand herangezogen bzw. wurde das KwEG als Instrument zur Implementierung einer neuen Staats- und Gesellschaftsordnung benutzt.

Welch große Aktivität die Regierung entwickelte, zeigt sich etwa daran, dass im Jahr 1922 mit

\footnotetext{
116 BGBl. 558/1933.

117 BGBl. I 208/1934.

118 BGB1. 280/1933.

${ }^{119}$ Nach der Regierungsumbildung im September 1933 hätte die notwendige Mehrheit im Nationalrat gefehlt.
}

rund 230 erlassenen Gesetzen der höchste Output in der Ersten Republik erfolgte. ${ }^{120}$ In den 14 Monaten des hier behandelten Zeitraumes wurden mehr als doppelt so viele "gesetzesgleiche" Verordnungen erlassen. Zwischen dem 7. März 1933 und dem 18. April 1934 tagte der Ministerrat $87 \mathrm{Mal}$, davon einige Male mehrtägig.

Die Ausdehnung der Verordnungen weit über den vom KwEG vorgegebenen Rahmen hinaus führte $\mathrm{zu}$ einer permanenten Demontage des Rechtsstaates.

Nach dem Ende des Zweiten Weltkrieges wurde die Verfassung „,nach dem Stande der Gesetzgebung vom 5. März 1933" wieder in Kraft gesetzt, ${ }^{121}$ die von März 1933 bis Ende April 1934 erlassenen Verordnungen wurden, soweit sie nicht verfassungswidrig waren, in den Rechtsbestand der Zweiten Republik übernommen. Einzelne blieben bis weit ins 20. Jahrhundert in Kraft. ${ }^{122}$

\footnotetext{
120 POLASCHEK, Rechtsentwicklung, 1.

${ }^{121}$ Verfassungs-Überleitungsgesetz vom 1. Mai 1945, StGBl 4/1945.

122 Die "Goldklauselverordnung, BGBl. 73/1933, wurde erst durch BGBl. I 125/1998 aufgehoben.
} 


\section{Korrespondenz:}

Prof. Dr. Martin F. POLASCHEK

Universität Graz

Universitätsplatz 3,

A-8010 Graz

martin.polaschek@uni-graz.at

ORCID-Nr. 0000-0003-3464-2637.

\section{Abkürzungen:}

DDSG Donau-Dampfschifffahrtsgesellschaft TOP Tagesordnungspunkt

Siehe auch das allgemeine Abkürzungsverzeichnis: [http://www.rechtsgeschichte.at/files/abk.pdf]

\section{Literatur:}

Kurt BAUER, Die österreichischen Anhaltelager 19331938 (2010), http://www.kurt-bauer-geschichte.at/ PDF_Forschung_Unterseiten/Anhaltelager.pdf (abgerufen 11. 3. 2018).

Eszter DORNER-BRADER (Bearb.), Protokolle des Ministerrates des Ersten Republik, Abt. VIII: Kabinett Dr. Engelbert Dollfuß, Bd. 4 (Wien 1984).

Gertrude ENDERLE-BuRCEL (Bearb.), Protokolle des Ministerrates des Ersten Republik, Abt. VIII: Kabinett Dr. Engelbert Dollfuß, Bde. 1-3, 5-7 (Wien 1980-1986).

Walter Goldinger (Hg.), Protokolle des Klubvorstandes der Christlichsozialen Partei 1932-1934 (Wien 1980).

Peter HuEmer, Sektionschef Robert Hecht und die Zerstörung der Demokratie in Österreich (Wien 1975).

Martin F. POLASCHEK, Die Rechtsentwicklung in der Ersten Republik. Die Gesetzgebung im Verfassungs- und Strafrecht von 1918-1933 (Graz 1992).

Martin F. POLASCHEK, Todesstrafe und Todesurteile in Österreich zwischen 1918 und 1938, in: Claudia Kuretsidis-Haider, Heimo Halbrainer, Elisabeth EBNER (Hgg.), Mit dem Tode bestraft. Historische und rechtspolitische Aspekte zur Todesstrafe in Österreich im 20. Jahrhundert und der Kampf um ihre weltweite Abschaffung (Graz 2008) 41-57.

Franz TIMISCHL, Beiträge zum steirischen Schulwesen von 1933 bis 1938 (Graz 1991).

Emmerich TALOS, Das austrofaschistische Herrschaftssystem. Österreich 1933-1938 (WienMünster 22013). 